For mat $i$ on of wave- front pat ter $n$ accompani ed by cur rent-dri ven el ectrost at i c i on- cycl ot ron i nst abi I i ti es

\begin{tabular}{|l|l|}
\hline $\begin{array}{l}\text { j our nal or } \\
\text { publ i cat i on ti t l e }\end{array}$ & Physi cs of pl asmas \\
\hline vol une & Vol . 4 \\
\hline number & No. 8 \\
\hline page r ange & pp. 2886- 2892 \\
\hline year & $1997-08-01$ \\
\hline URL & ht t p: //hdl . handl e. net /10655/3828 \\
\hline
\end{tabular}




\title{
Formation of wave-front pattern accompanied by current-driven electrostatic ion-cyclotron instabilities
}

\author{
Seiji Ishiguro \\ Department of Electronic Engineering, Tohoku University, Sendai 980-77, Japan
}

Tetsuya Sato, Hisanori Takamaru, Kunihiko Watanabe, and the Complexity Simulation Group ${ }^{\mathrm{a})}$

Theory and Computer Simulation Center, National Institute for Fusion Science, Nagoya 464-01, Japan

(Received 25 February 1997; accepted 15 May 1997)

\begin{abstract}
Formation of a wave-front pattern accompanied by an electrostatic ion-cyclotron instability driven by electrons drifting along a magnetic field is investigated by two-and-half dimensional particle simulations. A clear spatial wave-front pattern appears as the ion cyclotron wave grows due to the instability. When the electron stream is uniform in the system, an obliquely intersected stripe wave-front pattern is formed. When the stream has a bell-shaped pattern across the magnetic field, a V-shaped stripe wave-front pattern appears. The wave fronts have small angles with the magnetic field lines and propagate from the high-stream region to the low-stream region. (C) 1997 American Institute of Physics. [S1070-664X(97)03308-9]
\end{abstract}

\section{INTRODUCTION}

An electrostatic ion-cyclotron wave is destabilized by electrons drifting along a magnetic field. ${ }^{1}$ It is shown that electrostatic ion-cyclotron waves are destabilized more with lower electron drift velocity than the ion acoustic waves for a wide range of electron to ion temperature ratios. ${ }^{2}$

Oscillations around the ion-cyclotron frequency are observed in space observations. ${ }^{3,4}$ The ion conic formation associated with ion heating has been discussed in the connection with this instability.

In the laboratories, oscillations around the ion-cyclotron frequency have been extensively investigated in Q machines since the first observation of D'Angelo and Motley., ${ }^{5,6}$ The experimental results are said to be explained by the theory developed by Drummond and Rosenbluth. ${ }^{1}$ In the usual Q-machine experiments, a positive potential is applied to a small electrode in the plasma to induce an electric current, so the electric field has not only a component along the magnetic field lines, but also a component across the magnetic field lines. This has given rise to many arguments about the generation mechanism of the oscillation in Q-machine experiments. ${ }^{7-10}$

Particle simulations have disclosed the linear growth of the instability, saturation due to quasi-linear flattening of the electron velocity distribution and the ion perpendicular heating. ${ }^{11-13}$ However, only a few attempts ${ }^{14}$ are concerned with the pattern or structure formations associated with the current-driven electrostatic ion-cyclotron instability, although several extensive works have been done on the potential structure associated with the ion acoustic instability. ${ }^{15-17}$

In this work we would like to investigate the pattern formation associated with the current-driven electrostatic ion-cyclotron instability. In particular, we pay attention to the cases in which the electron drift velocity is smaller than the electron thermal velocity, whereas in the previous works,

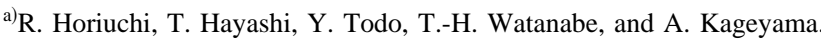

simulations ${ }^{11,13,14}$ have dealt with the cases in which the electron drift velocity is larger than the electron thermal velocity. We adopt a two-and-half dimensional electrostatic particle simulation with a periodic model and an external magnetic field in the $x-y$ plane.

A simulation model is described in Sec. II. Section III and Sec. IV present results of a uniform electron stream and a non-uniform electron stream, respectively. The summary and discussion are given in Sec. V.

\section{SIMULATION MODEL}

We employ a two-and-half dimensional electrostatic magnetized particle simulation with a periodic boundary model in which an external uniform magnetic field directs to the positive $x$ direction. We calculate ion velocities in three dimensions $\left(v_{x}, v_{y}, v_{z}\right)$ to follow the ion-cyclotron motion, while we adopt the guiding center drift approximation for electrons. In a two-dimensional model, the electric field has only $x$ and $y$ components, so that the particle $E \times B$ drift motion is pointing into the $z$ direction because the magnetic field directs in the $x$ direction in our configuration. Thus, we follow the electron motion only along the magnetic field ( $x$ direction). Electrons and ions are uniformly loaded in the system with $L_{x} \times L_{y}$ at $t=0$. The initial ion velocity distribution is a stationary Maxwellian and the initial electron velocity distribution is a shifted-Maxwellian with the drift speed $v_{d e}$ in the positive $x$ direction. As shown in Fig. 1, we present the results of two cases. In the first case the electron stream is uniform (a) in the system and is given by $v_{d e}=0.8 v_{t e}\left(=0.8\left(T_{e} / m_{e}\right)^{1 / 2}\right)$, where $v_{t e}, T_{e}$, and $m_{e}$ are electron thermal speed, electron temperature (energy unit), and electron mass, respectively. In the second case, it is nonuniform (b) in the $y$ direction and is given by $v_{d e}(y)=\left[0.6-0.2 \cos \left(2 \pi y / L_{y}\right)\right] v_{t e}$. The other simulation parameters are as follows. The ion to electron mass ratio $m_{i} / m_{e}$ is fixed to 400 . The ratio of the electron cyclotron to electron plasma frequency is $\omega_{c e} / \omega_{p e}=5$, and the ratio of the ion-cyclotron frequency to the electron plasma frequency is $\omega_{c i} / \omega_{p e}=0.0125$. The ion to electron temperature ratio is 
a)

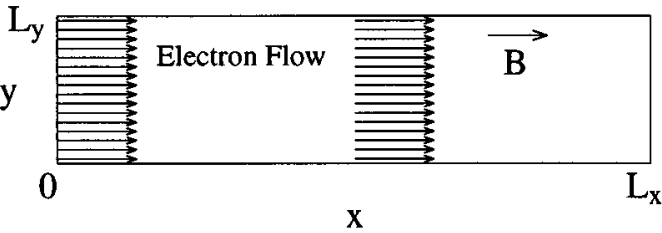

b)

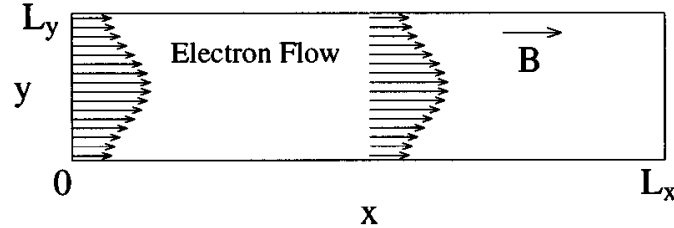

FIG. 1. Sketch of simulation system and initial electron stream profiles: uniform stream profile (a) and bell-shaped stream profile (b).

$T_{i} / T_{e}=0.5$. The time step width $\Delta t$ is $0.2 \omega_{p e}^{-1}$, where $\omega_{p e}$ is the electron plasma frequency. The system sizes $L_{x}$ and $L_{y}$ are $512 \lambda_{D e}$ and $128 \lambda_{D e}$, respectively, in the $x$ and $y$ directions, and $512 \times 128$ spatial grid system is used. Here, $\lambda_{D e}$ is the Debye length. The number of electrons and ions per unit cell is 256. Note that in these parameters the ion cyclotron wave is unstable and the ion acoustic wave is not unstable.

\section{UNIFORM ELECTRON STREAM}

Before presenting the simulation results, we briefly describe the dispersion relation for the electrostatic ion cyclotron wave in an infinite uniform plasma. The dispersion relation of electrostatic magnetized plasma is given by

$$
\begin{aligned}
0= & +\sum_{j=i, e} \sum_{n=-\infty}^{\infty} \frac{\Gamma_{n}\left(\mu_{j}\right)}{k^{2} \lambda_{D j}^{2}} \\
& \times\left[1+\frac{\omega-k_{\|} v_{d j}}{\sqrt{2} k_{\|} v_{t j}} Z\left(\frac{\omega-k_{\|} v_{d j}+n \omega_{c j}}{\sqrt{2} k_{\|} v_{t j}}\right)\right],
\end{aligned}
$$

where $v_{t j}=\left(T_{j} / m_{j}\right), v_{d j}$, and $\omega_{c j}$ are the thermal velocity, drift velocity and cyclotron frequency, respectively,
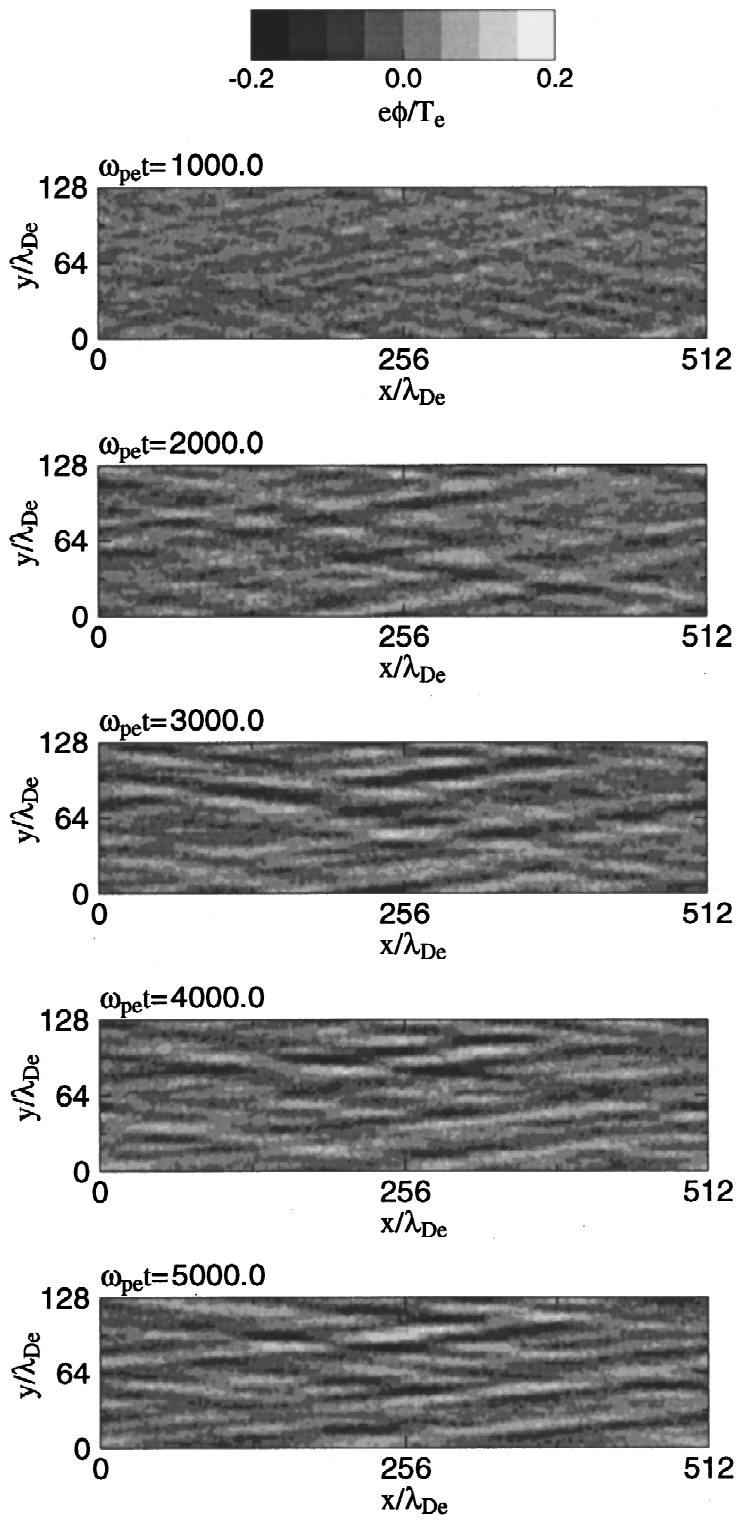

FIG. 2. Gray scale plot of the potential profile at $\omega_{p e} t=1000,2000,3000$, 4000 , and 5000 , for the case with uniform electron stream.

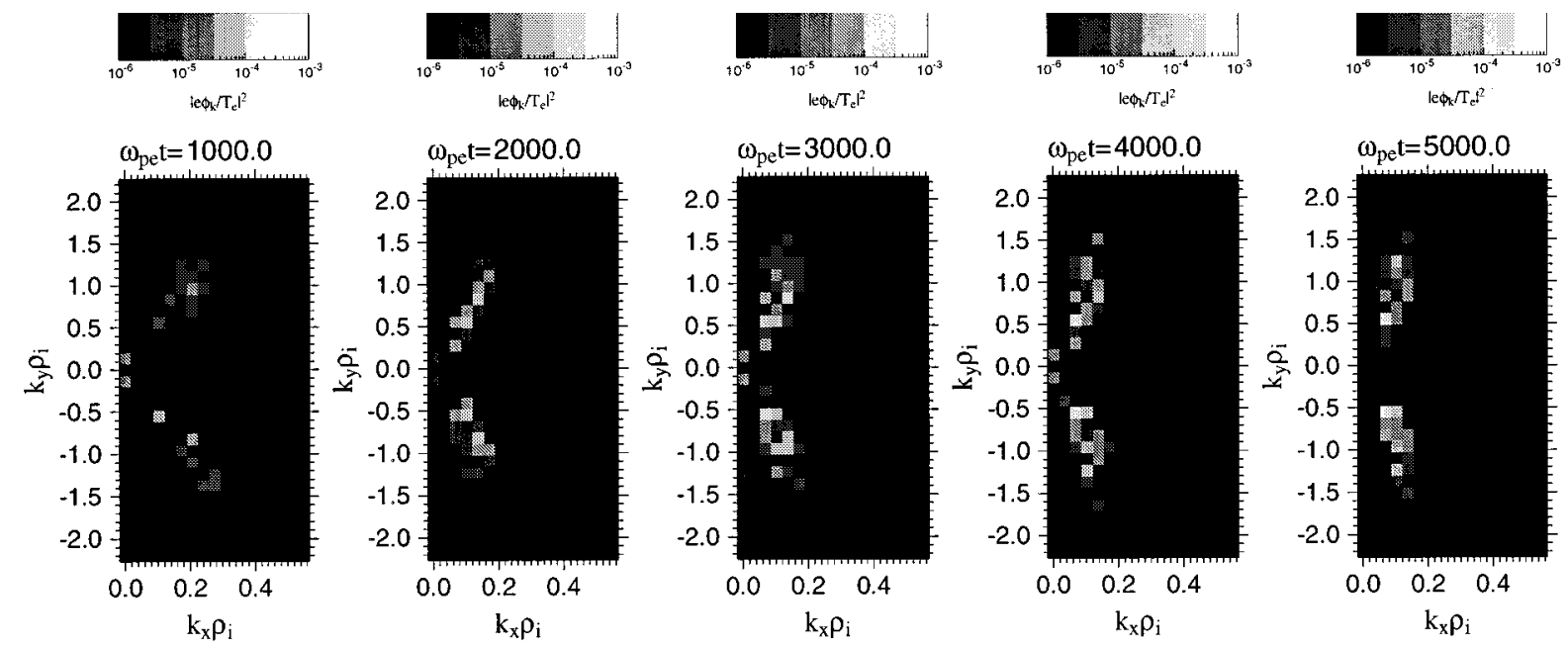

FIG. 3. Gray scale plot of the Fourier spectrum of the potential profile at $\omega_{p e} t=1000,2000,3000,4000$, and 5000, for the case with uniform electron stream. 
a)

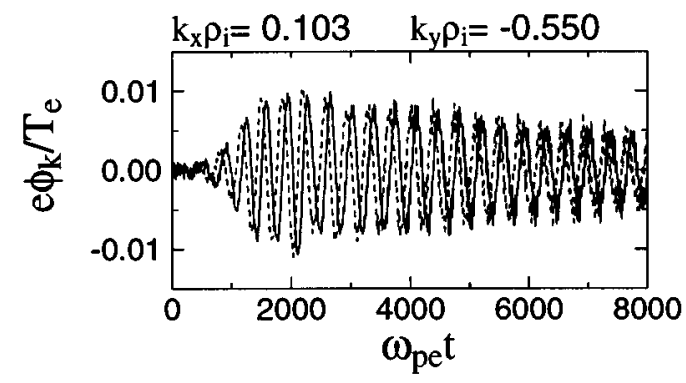

b)

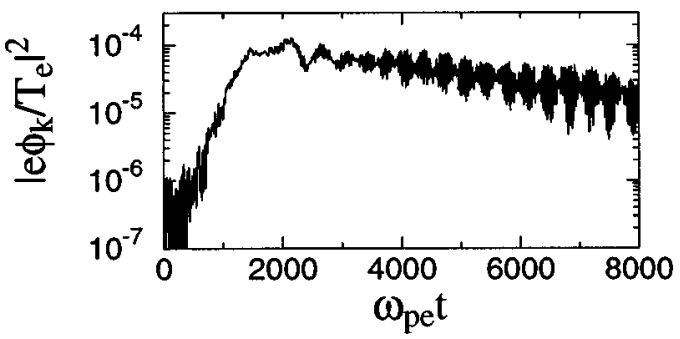

c)

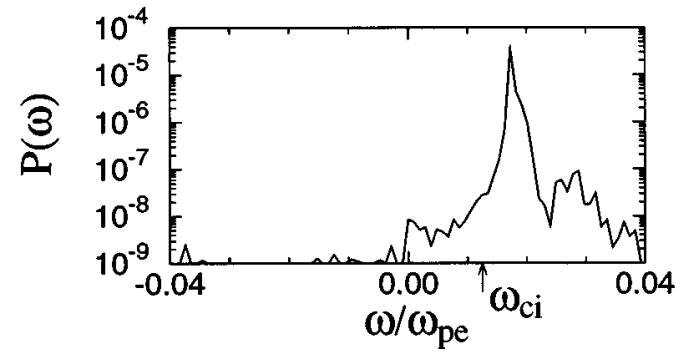

FIG. 4. Time evolutions of the real (solid line) and the imaginary part (dashed line) (a), the square of the absolute value (b), and the frequency spectrum of $(3,-4)$ mode of the potential with $k_{x} \rho_{i}=0.103, k_{y} \rho_{i}=-0.55$ for the case with uniform electron stream.

$\Gamma_{n}\left(\mu_{j}\right)=\exp \left(-\mu_{j}\right) I_{n}\left(\mu_{j}\right), \mu_{j}=\left(k_{\perp} v_{t j} / \omega_{c j}\right)^{2} \equiv\left(k_{\perp} \rho_{j}\right)^{2}, I_{n}$ is the modified Bessel function of order $n, \rho_{j}=\left(T_{j} / m_{j} \omega_{j}^{2}\right)^{1 / 2}$ is the gyroradius, $\lambda_{D j}\left(=v_{t j} / \omega_{p j}\right)$ is the Debye length, $Z$ is the plasma dispersion function, $k_{\|}$and $k_{\perp}$ are parallel and perpendicular wave number with respect to the magnetic field, respectively, and $\omega$ is the complex frequency. The numerical solution of this equation for the simulation parameter of uniform electron stream shows that the fundamental mode of the ion cyclotron wave with the real frequency $\omega_{r} \sim \omega_{c i}$ is unstable where $0.08 \leqq k_{\|} \rho_{i} \leqq 0.2$ and $0.4 \leq k_{\perp} \rho_{i} \lesssim 1.5$, and the second harmonics with $\omega_{r} \sim 2 \omega_{c i}$ is unstable where $0.11 \lesssim k_{\|} \rho_{i} \leqslant 0.18$ and $1.0 \leqslant k_{\perp} \rho_{i} \lesssim 1.6$. On the other hand, the ion acoustic wave is not unstable.

Here, we show the results of the first case where the initial electron drift is spatially uniform and given by $v_{d e}=0.8 v_{t e}$. Figure 2 shows the gray scale plots of potential profiles at $\omega_{p e} t=1000,2000,3000,4000$, and 5000, which are averaged over the three times of the plasma period $3 \times 2 \pi / \omega_{p e}$. Small scale fluctuations observed at $\omega_{p e} t=1000$ grow with time, and an obliquely intersected stripe pattern appears at $\omega_{p e} t=2000$. The pattern is composed of lines having an angle of \pm 10 degrees with respect to the magnetic filed lines. The angle between the lines of the pattern and the magnetic field lines decreases with time from $\omega_{p e} t=2000$ to 5000 . The pattern observed at $\omega_{p e} t=5000$ is a)

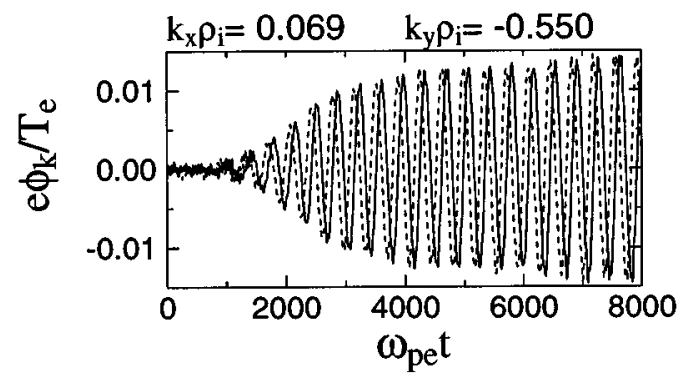

b)

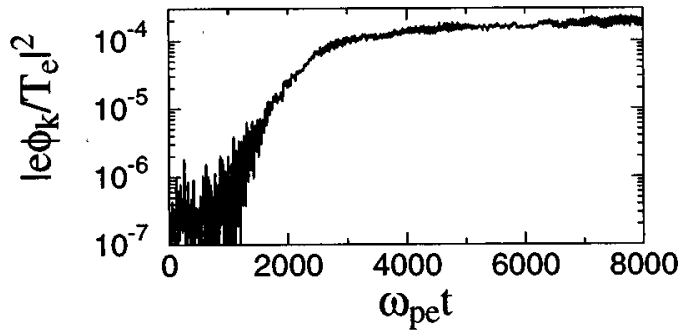

c)

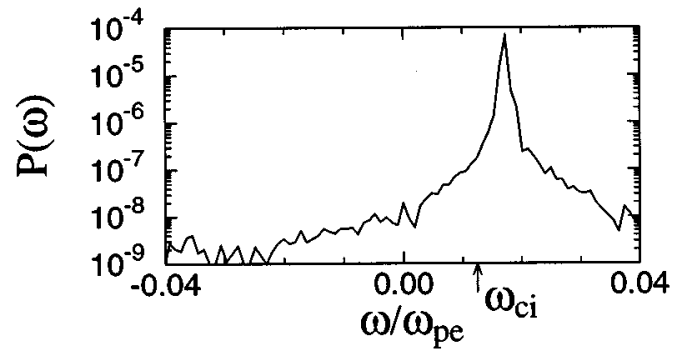

FIG. 5. Time evolutions of the real (solid line) and the imaginary part (dashed line) (a), the square of the absolute value (b), and the frequency spectrum of $(2,-4)$ mode of the potential with $k_{x} \rho_{i}=0.069, k_{y} \rho_{i}=-0.55$ for the case with uniform electron stream.

composed of the lines with an angle of \pm 7 degrees with respect to the magnetic field lines. As shown later, the pattern corresponds to the electrostatic ion cyclotron waves propagating in the direction of \pm 83 degrees with respect to the magnetic field lines.

To see the spatial Fourier modes corresponding to the pattern, we show the gray scale plots of the spatial Fourier mode distributions of potential profiles in Fig. 3. Initially some modes with $k_{\|} / k_{\perp}\left(=\left|k_{x} / k_{y}\right|\right) \simeq 0.2$ and $0.1 \lesssim k_{\|} \rho_{i}\left(=k_{x} \rho_{i}\right) \lessgtr 0.3$ are appearing. At $\omega_{p e} t=2000$ the modes $(3, \pm 4)$ with $k_{\|} \rho_{i} \simeq 0.1$ and $k_{\perp} \rho_{i} \simeq 0.55$ are large. This corresponds to the pattern appearing in the potential profile at $\omega_{p e} t=2000$. The modes with smaller $k_{\|}$grow from $\omega_{p e} t=2000$ to 4000 , while the modes with $k_{\|} \rho_{i} \geqslant 0.2$ disappear. This modification of the mode pattern is consistent with the result that the angle between the equi-potential lines and the magnetic field lines decreases with time.

Figure 4 shows time evolutions of $(3,-4)$ mode (a) of the potential with $k_{x} \rho_{i}=0.103, k_{y} \rho_{i}=-0.55$, where the solid line and the dashed line indicate the real and the imaginary part, respectively, the square of the absolute value (b), and the frequency spectrum (c). This mode is unstable to the current driven electrostatic ion cyclotron instability for the initial condition. The frequency spectrum has a peak value at $\omega / \omega_{c i} \simeq 1.4$, which is consistent with the linear dispersion relation. This mode represents the wave propagating to the 
a)

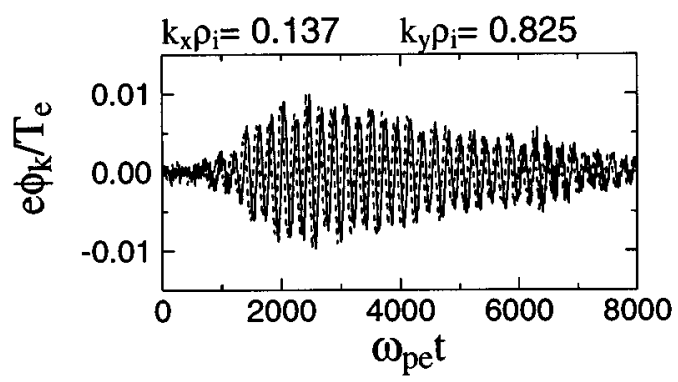

b)

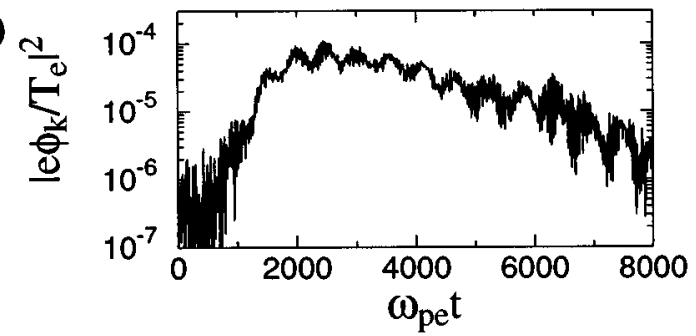

c)

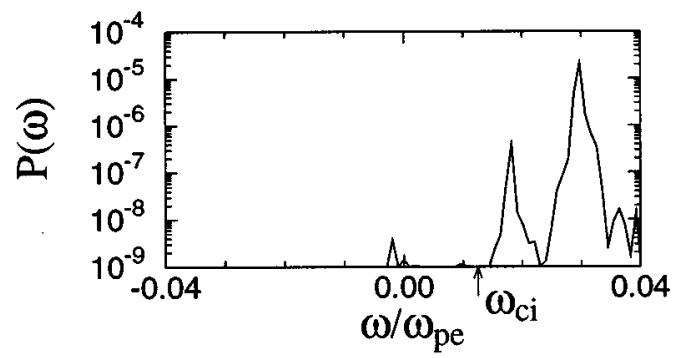

FIG. 6. Time evolutions of the real (solid line) and the imaginary part (dashed line) (a), the square of the absolute value (b), and the frequency spectrum of $(4,6)$ mode of the potential with $k_{x} \rho_{i}=0.137, k_{y} \rho_{i}=-0.825$ for the case with uniform electron stream.

direction with an angle of -79 degrees with respect to the magnetic field. The parallel component of the phase velocity is $\omega / k_{\|} \simeq 0.48 v_{t e}$. The amplitude increases from $\omega_{p e} t \simeq 500$ to 1500 , experiencing saturation around $\omega_{p e} t \simeq 1500$. Thereafter, the amplitude decreases gradually. In Fig. 5, we show the time evolutions of $(2,-4)$ mode (a) of the potential with $k_{x} \rho_{i}=0.069, k_{y} \rho_{i}=-0.55$, the square of the absolute value (b), and the frequency spectrum (c). There is a peak at $\omega / \omega_{c i} \simeq 1.4$ in the frequency spectrum as was observed in the mode $(3,-4)$. This mode represents the wave propagating to the direction with -83 degrees with respect to the magnetic field and the parallel component of the phase velocity is $\omega / k_{\|} \simeq 0.71$, which is larger than that of the mode $(3,-4)$. The amplitude starts increasing at $\omega_{p e} t \simeq 1000$, which is followed by a rapid increase up to $\omega_{p e} t \simeq 2500$. After $\omega_{p e} t \simeq 3000$, the growth is almost saturated. Though this mode has a small growth rate at the initial condition, it has a rapid increase of the amplitude. This is caused by the fact that the electron velocity distribution has a steep slope around the phase velocity of this mode. This modification of the electron velocity distribution is caused by the velocity space diffusion. Figure 6 shows the time evolutions of $(4,6)$ mode (a) of the potential with $k_{x} \rho_{i}=0.137$, $k_{y} \rho_{i}=0.825$, the square of the absolute value (b), and the frequency spectrum (c). There are two peaks; a large peak

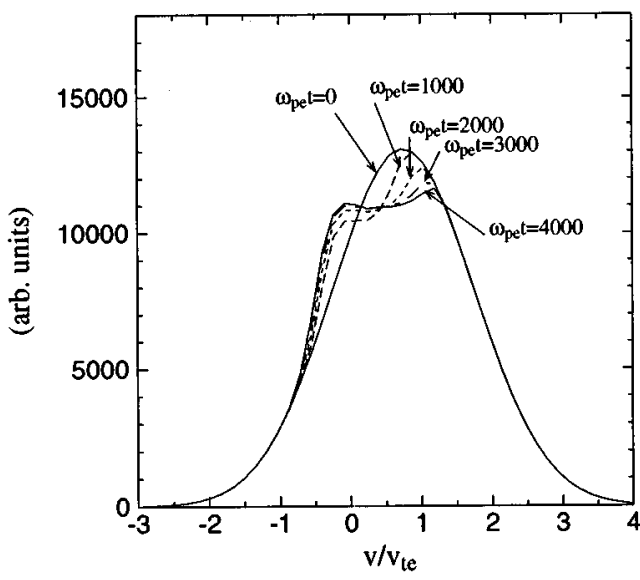

FIG. 7. Electron velocity distribution at $\omega_{p e} t=1000,2000,3000,4000$, and 5000 for the case with uniform electron stream.

around $\omega / \omega_{c i} \simeq 2.4$, which corresponds to the second harmonics of the ion-cyclotron wave, and a small peak around $\omega / \omega_{c i} \simeq 1.4$, which corresponds to the fundamental mode. The propagation angle of this mode with respect to the magnetic field is 81 degrees and the parallel component of the phase velocity for the second harmonics is $\omega / k_{\|} \simeq 0.61 v_{t e}$. The amplitude starts increasing at $\omega_{p e} t \simeq 700$, which is followed by a rapid increase up to $\omega_{p e} t \simeq 2000$. After peaking at $\omega_{p e} t \simeq 2000$, it starts decreasing.

It is interesting to observe the modification of the electron velocity distribution in connection with the wave-front pattern. In Fig. 7, we show the electron velocity distributions along the magnetic field at $\omega_{p e} t=0,1000,2000,3000$, and 4000. The velocity space diffusion gives rise to a change of the steepest region in the velocity distribution. At $\omega_{p e} t=1000$, the velocity distribution has a positive steepest gradient around $v / v_{t e}=0.5$, which is located in the vicinity of the parallel component of the phase velocity of the mode $(2,-4)$. This is consistent with the rapid growth of mode $(2,-4)$ at $\omega_{p e} t=1000$. The region of steepest gradient moves to the higher velocity region with time and the velocity distribution becomes flattened in the $-0.5<v / v_{t e}<1.0$.

Figure 8 shows time evolution of the ion perpendicular kinetic energy. This starts to increase at $\omega_{p e} t \simeq 500$, which is followed by a rapid increase up to $\omega_{p e} t \simeq 2000$. A gradual increase proceeds to $\omega_{p e} t \simeq 4000$ and saturates. This satura-

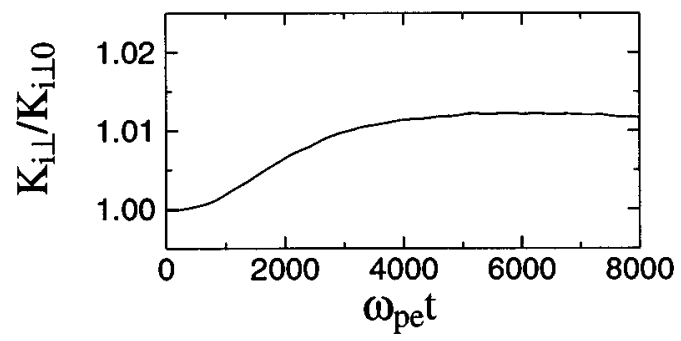

FIG. 8. Time evolution of the ion perpendicular kinetic energy for the case with uniform electron stream. 

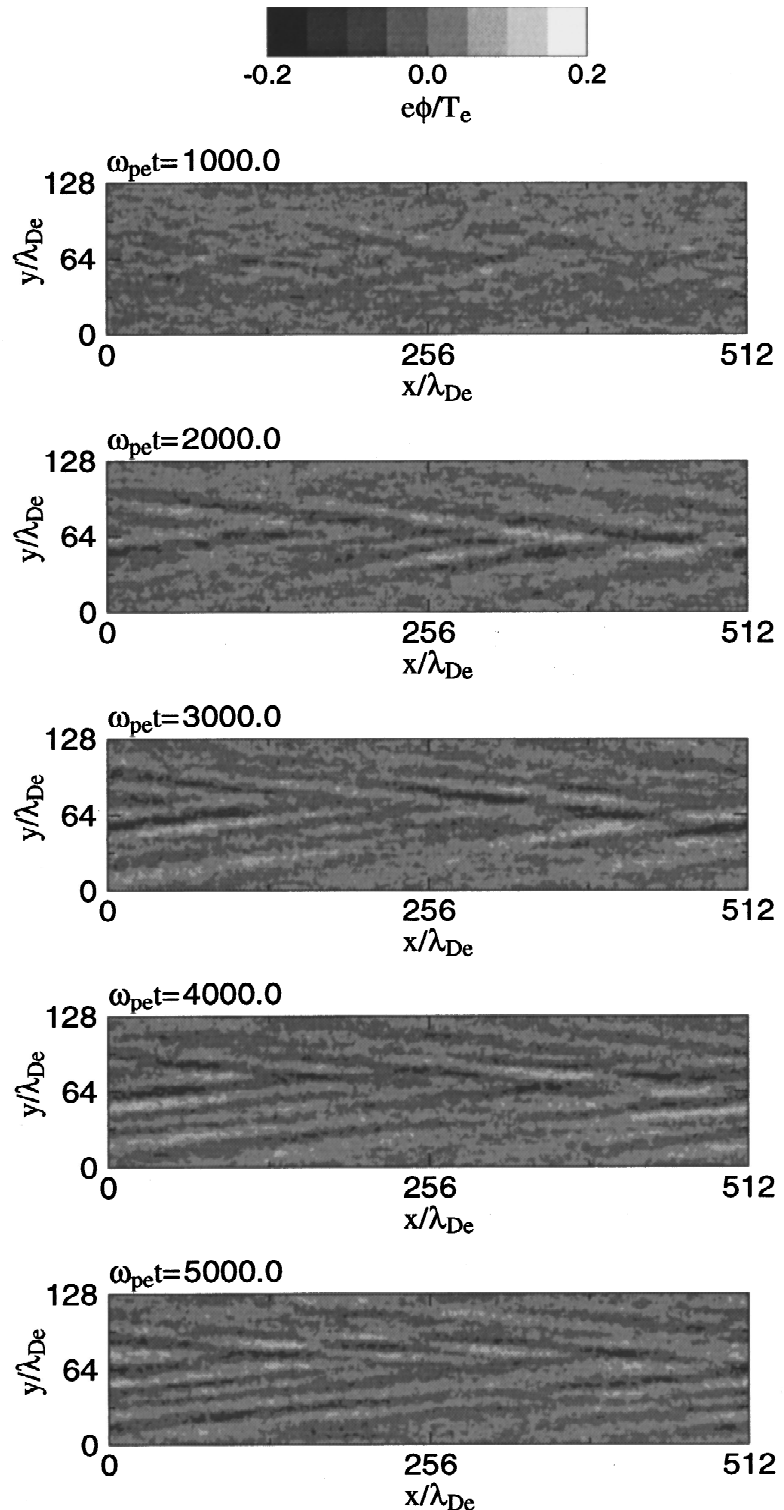

FIG. 9. Gray scale plot of the potential profile at $\omega_{p e} t=1000,2000,3000$, 4000, and 5000 for the case with bell-shaped electron stream. tion corresponds to the saturation of the instability due to the flattening of the electron velocity distribution. The increase of the ion perpendicular kinetic energy remains in the level with about $1 \%$.

\section{BELL-SHAPED ELECTRON STREAM}

In this section we present the results of the second simulation run where the electron stream is bell-shaped in the $y$ direction. Note that the averaged electron drift velocity is smaller than that of the previous case. Figure 9 shows the gray scale plot of potential profiles at $\omega_{p e}=1000,2000$, 3000 , 4000, and 5000, which are averaged over the three times of the plasma period $3 \times 2 \pi / \omega_{p e}$. We can see a clear $\mathrm{V}$-shaped stripe pattern at $\omega_{p e} t=2000$. In the upper half plane, the equi-valued lines lean to the right, whereas they lean to the left in the lower half plane. The angle between the equi-potential lines and the magnetic field lines is about \pm 10 degrees. The angle decreases with time and becomes about \pm 7 degrees at $\omega_{p e} t=5000$. In Fig. 10, we show the gray scale plots of the spatial Fourier mode distributions of the potential profiles. Initially some modes with $k_{\|} / k_{\perp}\left(=\left|k_{x} / k_{y}\right|\right) \simeq 0.2$ and $0.1 \lesssim k_{\|} \rho_{i}\left(=k_{x} \rho_{i}\right) \lesssim 0.2$ are appearing. At $\omega_{p e} t=2000$, the modes $(3, \pm 4)$ with $k_{\|} \rho_{i} \simeq 0.1$ and $k_{\perp} \rho_{i} \simeq 0.55$ and the mode $(3, \pm 5)$ with $k_{\|} \rho_{i} \simeq 0.1$ and $k_{\perp} \rho_{i} \simeq 0.69$ are dominant. The mode $(3,4)$ corresponds to the pattern in the upper half plane, whereas the mode $(3,-4)$ corresponds to the pattern in the lower half plane. The modes with smaller $k_{\|}$grow from $\omega_{p e} t=1000$ to 5000, and the mode with $k_{\|} \rho_{i}=0.07$ becomes the dominant mode at $\omega_{p e} t=5000$. The mode $(2,4)$ corresponds to the pattern in the upper half plane, whereas the mode $(2,-5)$ corresponds to the lower half plane. This modification of the mode pattern is consistent with the result that the angle between the equi-potential lines and the magnetic field lines decreases with time.

To examine the time evolution of the pattern in a short time scale, we show the gray scale plot of the potential profile from $\omega_{p e} t=3000$ to 3150 , in Fig. 11. We can see the
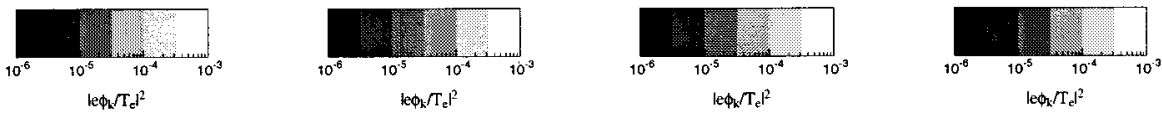

$\operatorname{le}_{\mathrm{k}} / \mathrm{T}_{\mathrm{c}} \mathrm{l}^{2}$
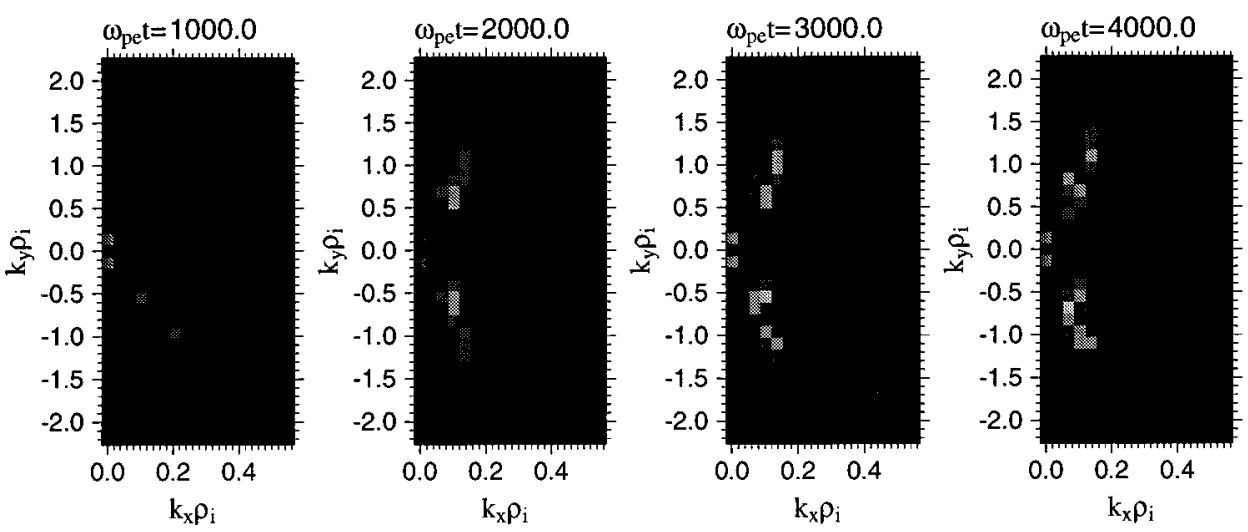

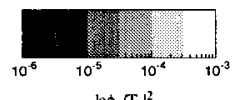

$\mathrm{le} \phi_{\mathrm{k}} \mathrm{T}_{\mathrm{c}} \mathrm{l}^{2}$

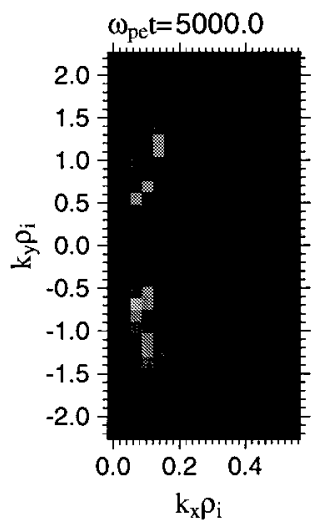

FIG. 10. Gray scale plot of the Fourier spectrum of the potential profile at $\omega_{p e} t=1000,2000,3000$, 4000, and 5000 for the case with bell-shaped electron stream. 

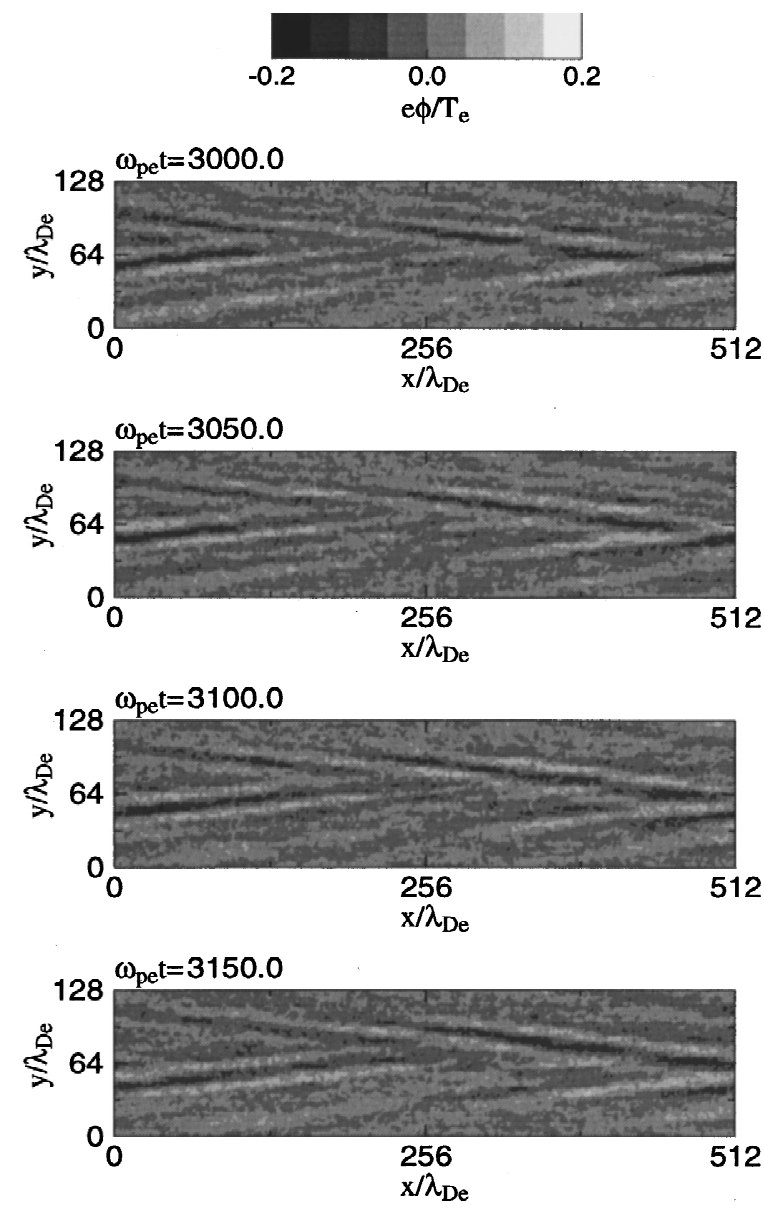

FIG. 11. Gray scale plot of the potential profile at $\omega_{p e} t=3000,3050,3100$, and 3150 for the case with bell-shaped electron stream.

propagation of waves from the high electron stream region to the low electron stream region. In the upper half plane, the wave is propagating with an angle with 80 degrees with respect to the magnetic field lines, whereas in the lower half plane the wave is propagating with an angle with -80 degrees with respect to the magnetic field lines. The wavelength is about $30 \lambda_{D e}\left(=11 \rho_{i}\right)$ and the propagation speed is about $0.1 v_{t e}$. These results indicate that the ion-cyclotron waves excited in the high electron stream region propagate to the low electron stream region. The wave in the upper half plane is considered mainly to correspond to the $(3,4)$ mode, while the wave in the lower half plane is considered mainly to correspond to the $(3,-4)$ mode.

Figure 12 shows time evolutions of the mode $(3,-4)$ (a) of the potential with $k_{x} \rho_{i}=0.103, k_{y} \rho_{i}=-0.55$, where the solid line and dashed line indicate the real and the imaginary part, respectively, the square of the absolute value (b), and the frequency spectrum (c). The amplitude starts increasing at $\omega_{p e} t \simeq 500$, attaining to a maximum value at $\omega_{p e} t \simeq 3500$. After that, it gradually decreases. The growth rate is smaller than that in the previous case because the averaged electron drift speed is smaller than that of the previous case. The real frequency is $\omega_{r} \simeq 1.4 \omega_{c i}$, which corresponds to the fundamental mode of the ion-cyclotron wave. This mode corresponds to the wave pattern shown in Fig. 11. Figure 13 shows the time evolution of the mode $(2,-5)$ (a) a)

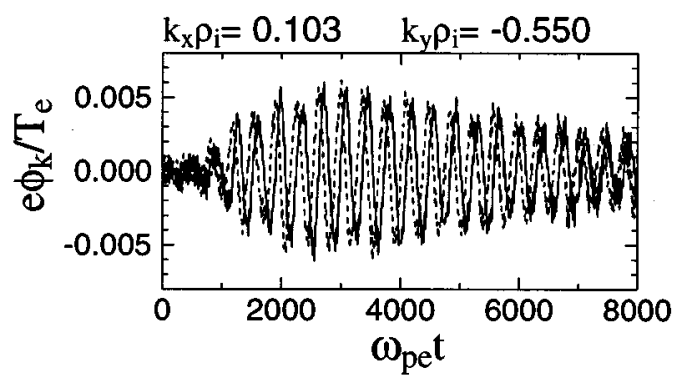

b)

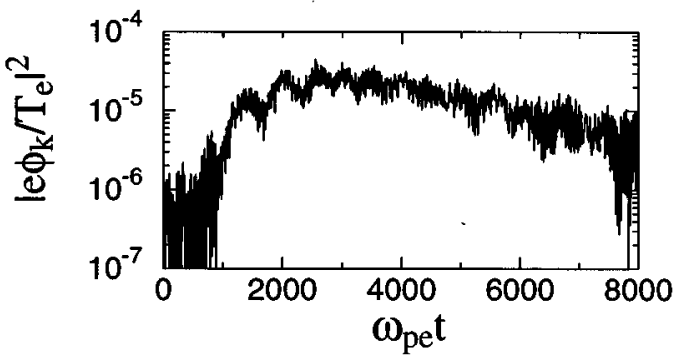

c)

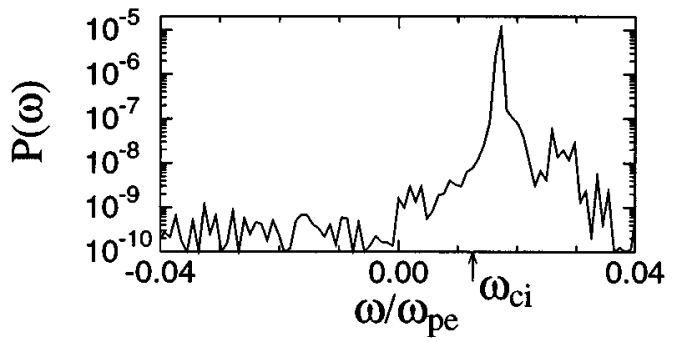

FIG. 12. Time evolutions of the real (solid line) and the imaginary part (dashed line) (a), the square of the absolute value (b), and the frequency spectrum of $(3,-4)$ mode of the potential with $k_{x} \rho_{i}=0.103, k_{y} \rho_{i}=-0.55$ for the case with bell-shaped electron stream.

of the potential with $k_{x} \rho_{i}=0.07$ and $k_{y} \rho_{i}=-0.69$, where the solid line and dashed line indicate the real and the imaginary part, respectively, the square of the absolute value (b), and the frequency spectrum (c). The amplitude starts growing at $\omega_{p e} t \simeq 2000$, reaching a maximum at $\omega_{p e} t \simeq 4500$. Thereafter it gradually decreases up to $\omega_{p e} t \simeq 7000$, and increases again. The real frequency is also corresponding to the fundamental mode of the ion-cyclotron wave, which is one of the dominant mode around $\omega_{p e} t=4000$.

\section{SUMMARY AND DISCUSSION}

We have studied formation of wave-front patterns associated with the current driven electrostatic ion-cyclotron instability by means of a two-and-half dimensional electrostatic particle simulation. As the electrostatic ion-cyclotron wave grows, a clear wave-front pattern appears. When the initial electron stream is spatially uniform, an obliquely intersected stripe wave-front pattern appears. The angle between the equi-potential lines and the magnetic field lines decreases with time. As the ion-cyclotron wave grows, the electron velocity distribution along the magnetic field is modified by the velocity space diffusion, and as a result, the initial growing mode saturates and the modes with higher phase velocity (smaller $k_{\|}$) arise. ${ }^{11}$ This causes the decrease of angle between the equipotential lines and the magnetic field lines. 
a)

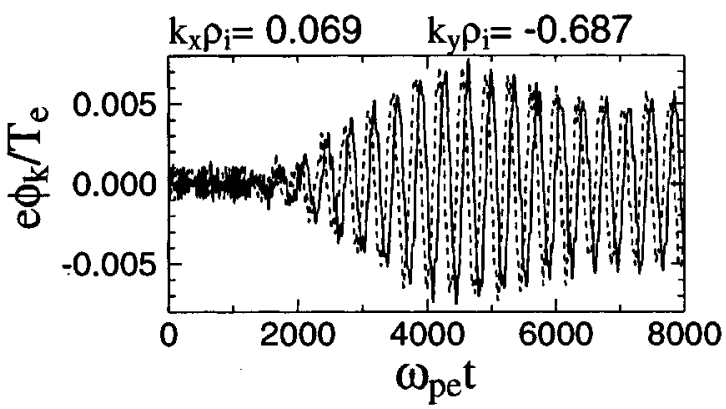

b)

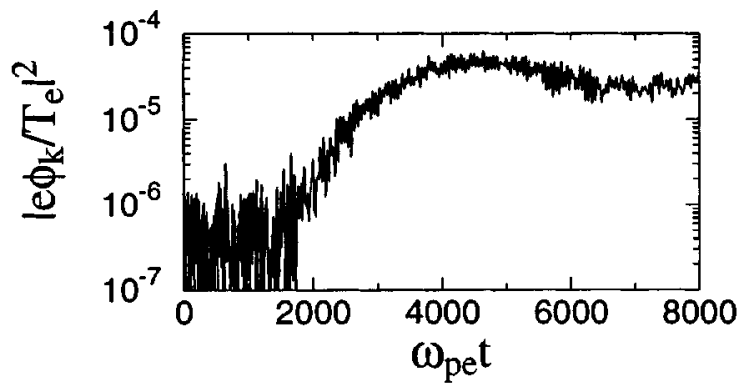

c)

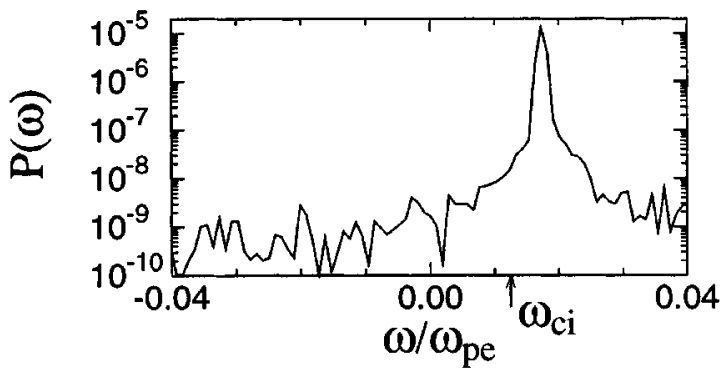

FIG. 13. Time evolutions of the real (solid line) and the imaginary part (dashed line) (a), the square of the absolute value (b), and the frequency spectrum of $(2,-5)$ mode of the potential with $k_{x} \rho_{i}=0.07, k_{y} \rho_{i}=-0.69$ for the case with bell-shaped electron stream.

When the electron stream is bell-shaped in the $y$ direction, a V-shaped stripe wave-front pattern appears. This is composed of the ion-cyclotron waves which are excited in the high stream region and propagate from the high-stream region to the low-stream region. The decrease of the angle between the equi-potential lines and the magnetic field lines is also observed in this case.

Kan and Okuda ${ }^{14}$ performed a two-dimensional simulation where the electron drift was non-uniform in the plane perpendicular to the magnetic field. In this work the electrostatic ion cyclotron instability was observed to cause striations in the plasma density, field aligned current, and electron energy flux. They were ascribed to nonlinear couplings among the electrostatic ion-cyclotron waves, which are related to the electron $E \times B$ drift. In their simulation model, the magnetic field is almost perpendicular to $x-y$ plane, and thus, electron $E \times B$ drift is retained. However, perturbations along the magnetic field are ignored. On the other hand, the magnetic field is in the $x-y$ plane in our model, and thus perturbations with any angles with respect to the magnetic field are retained. We have, thereby, observed the clear mode conversion to small $k_{\|}$modes. However, the perturbations accompanied by the $E \times B$ particle motion are ignored.
In the previous simulation ${ }^{11}$ a large ion perpendicular heating has been observed for the case in which the electron drift speed is larger than the electron thermal velocity. Furthermore, the larger ion heating and the ion conic formation also have been observed by further growth of the instability in the recycling model. ${ }^{12,14}$ On the other hand, the heating observed in the present simulation is quite small because the electron drift speed is small and no fresh drifting electrons are supplied. It is interesting to study whether a large ion perpendicular heating and resulting ion conic formation are observed or not by introducing fresh streaming electrons even when the electron drift speed is smaller than the electron thermal velocity.

In the present periodic boundary simulation, the growth of the wave amplitude saturates at a low level and no observable dc potential structure is created. Satellite observations have suggested that a V-shaped dc potential structure and electrostatic ion-cyclotron wave are simultaneously present above the auroral ionosphere. ${ }^{4}$ In this connection it is interesting to predict that further growth of the ion-cyclotron wave due to the injection of fresh electrons gives rise to a dc potential structure for the case in which the electron drift velocity is smaller than the electron thermal velocity. This will be published separately.

\section{ACKNOWLEDGMENTS}

One of the authors (S. I.) would like to thank Professor Noriyoshi Sato for his encouragement. The computations were performed at the Advanced Computing System for Complexity Simulation at the National Institute for Fusion Science and partially performed at the Computer Center of Tohoku University.

${ }^{1}$ W. E. Drummond and M. N. Rosenbluth, Phys. Fluids 5, 1507 (1962).

${ }^{2}$ J. M. Kindel and C. F. Kennel, J. Geophys. Res. 76, 3055 (1971).

${ }^{3}$ P. M. Kintner, M. C. Kelley, R. D. Sharp, A. G. Ghielmetti, M. Temerin, C. Cattell, P. F. Mizera, and J. F. Fennell, J. Geophys. Res. 84, 7201 (1979).

${ }^{4}$ F. S. Mozer, C. W. Carlson, M. K. Hudson, R. B. Torbert, B. Parady, J. Yatteau, and M. C. Kelley, Phys. Rev. Lett. 38, 292 (1977).

${ }^{5}$ N. D'Angelo and R. W. Motley, Phys. Fluids 5, 633 (1962); R. W. Motley and N. D'Angelo, ibid. 6, 296 (1963).

${ }^{6}$ N. Rynn, D. R. Dakin, D. L. Correll, and G. Benford, Phys. Rev. Lett. 33, 765 (1974); G. Benford, N. Rynn, J. J. Thomson, and W. S. Williamson, Phys. Fluids 17, 1001 (1974); D. L. Correll, N. Rynn, and H. Böhmer, ibid. 18, 1800 (1975); R. A. Stern, D. L. Correll, H. Böhmer, and N. Rynn, Phys. Rev. Lett. 37, 833 (1976); H. Böhmer and S. Fornaca, J. Geophys. Res. 84, 5234 (1979); N. S. Wolf, R. Majeski, H. Lashinsky, V. Tripathi, and C. S. Liu, Phys. Rev. Lett. 45, 799 (1980).

${ }^{7}$ N. Sato and R. Hatakeyama, J. Phys. Soc. Jpn. 54, 1661 (1985).

${ }^{8}$ R. Hatakeyama, F. Muto, and N. Sato, Jpn. J. Appl. Phys. 24, L285 (1985).

${ }^{9}$ J. J. Rasmussen and R. W. Schrittwieser, IEEE Trans. Plasma Sci. 19, 457 (1991).

${ }^{10}$ S. Ishiguro, Y. Suzawa, and N. Sato, J. Phys. Soc. Jpn. 63, 3651 (1994).

${ }^{11}$ H. Okuda, C. Z. Chen, and W. W. Lee, Phys. Fluids 24, 1060 (1981).

${ }^{12}$ H. Okuda and M. Ashour-Abdalla, Geophys. Res. Lett. 8, 811 (1981).

${ }^{13}$ P. L. Prichett, M. Ashour-Abdalla, and J. M. Dawson, Geophys. Res. Lett. 8, 611 (1981).

${ }^{14}$ J. R. Kan and H. Okuda, J. Geophys. Res. 88, 6339 (1983).

${ }^{15}$ T. Sato and H. Okuda, Phys. Rev. Lett. 44, 740 (1980); J. Geophys. Res. 86, 3357 (1981).

${ }^{16}$ C. Barnes, M. K. Hudson, and W. Lotko, Phys. Fluids 28, 1055 (1985).

${ }^{17}$ T. Sato, H. Takamaru, and the Complexity Simulation Group, Phys. Plasmas 2, 3609 (1995). 